\title{
Quantitative Imaging and Radiomics in Multiple Myeloma: A Potential Opportunity?
}

\author{
Alberto Stefano Tagliafico ${ }^{1,2, *}$, Alida Dominietto ${ }^{1}$, Liliana Belgioia ${ }^{1,2}{ }^{(}$, Cristina Campi $^{3}{ }^{\circledR}$, Daniela Schenone ${ }^{3}$ \\ and Michele Piana ${ }^{3,4}$ \\ 1 Department of Health Sciences (DISSAL), University of Genoa, 16129 Genoa, Italy; \\ alida.dominietto@hsanmartino.it (A.D.); liliana.belgioia@unige.it (L.B.) \\ 2 IRCCS Ospedale Policlinico San Martino, 16129 Genoa, Italy \\ 3 Department of Mathematics (DIMA), University of Genoa, 16129 Genoa, Italy; campi@dima.unige.it (C.C.); \\ daniela.schenone25@gmail.com (D.S.); piana@dima.unige.it (M.P.) \\ 4 CNR-SPIN, 16129 Genoa, Italy \\ * Correspondence: alberto.tagliafico@unige.it
}

Citation: Tagliafico, A.S.;

Dominietto, A.; Belgioia, L.; Campi,

C.; Schenone, D.; Piana, M.

Quantitative Imaging and Radiomics in Multiple Myeloma: A Potential Opportunity?. Medicina 2021, 57, 94. https://doi.org/10.3390/medicina 57020094

Academic Editor: Vincenzo Desiderio

Received: 7 January 2021

Accepted: 19 January 2021

Published: 21 January 2021

Publisher's Note: MDPI stays neutral with regard to jurisdictional claims in published maps and institutional affiliations.

Copyright: (c) 2021 by the authors. Licensee MDPI, Basel, Switzerland. This article is an open access article distributed under the terms and conditions of the Creative Commons Attribution (CC BY) license (https:/ / creativecommons.org/licenses/by/ $4.0 /)$.

\begin{abstract}
Multiple Myeloma (MM) is the second most common type of hematological disease and, although it is rare among patients under 40 years of age, its incidence rises in elderly subjects. MM manifestations are usually identified through hyperCalcemia, Renal failure, Anaemia, and lytic Bone lesions (CRAB). In particular, the extent of the bone disease is negatively related to a decreased quality of life in patients and, in general, bone disease in MM increases both morbidity and mortality. The detection of lytic bone lesions on imaging, especially computerized tomography (CT) and Magnetic Resonance Imaging (MRI), is becoming crucial from the clinical viewpoint to separate asymptomatic from symptomatic MM patients and the detection of focal lytic lesions in these imaging data is becoming relevant even when no clinical symptoms are present. Therefore, radiology is pivotal in the staging and accurate management of patients with MM even in early phases of the disease. In this review, we describe the opportunities offered by quantitative imaging and radiomics in multiple myeloma. At the present time there is still high variability in the choice between various imaging methods to study MM patients and high variability in image interpretation with suboptimal agreement among readers even in tertiary centers. Therefore, the potential of medical imaging for patients affected by MM is still to be completely unveiled. In the coming years, new insights to study MM with medical imaging will derive from artificial intelligence (AI) and radiomics usage in different bone lesions and from the wide implementations of quantitative methods to report CT and MRI. Eventually, medical imaging data can be integrated with the patient's outcomes with the purpose of finding radiological biomarkers for predicting the prognostic flow and therapeutic response of the disease.
\end{abstract}

Keywords: multiple myeloma; computed tomography; artificial intelligence; radiomics; prognosis; imaging; diagnosis

\section{Introduction}

Multiple Myeloma (MM) is the second most common type of hematological disease and, although it is rare among patients under 40 years of age, its incidence rises in elderly subjects [1]. This hematological malignant disease is characterized by the autonomous monoclonal proliferation of plasma cells in the bone marrow [2]. More specifically, MM is a cytogenetically heterogeneous disorder of clonal plasma cells in which an excessive production of either monoclonal intact immunoglobulin molecules or immunoglobulin free light chains kappa or lambda is crucial for the development of clinical features [3-7]. Many risk factors for $\mathrm{MM}$ are known, including male sex, radiation exposure and monoclonal gammopathy of undetermined significance (MGUS) $[1,6,8]$. 
MM manifestations are usually identified through hyperCalcemia, Renal failure, Anaemia, and lytic Bone lesions (CRAB). In particular, in MM the normal myeloproliferation is replaced by a pathological one, with important clinical impacts leading to an increasing risk of pathological fractures [2]. This is the main reason why, in 2003, the International Myeloma Working Group (IMWG) replaced the Durie-Salmon system with a revised version (Durie-Salmon system plus), where the diagnostic role of radiography in identifying bone marrow involvement is overtaken by the increased sensitivity of Magnetic Resonance Imaging (MRI) and hybrid Positron Emission Tomography (PET) and Computerized Tomography (CT) data with FDG as tracer (FDG PET/CT) [7,9,10]. Indeed, the extent of the bone disease is negatively related to a decreased quality of life in patients and, in general, bone disease in MM increases both morbidity and mortality [2,4,11-13]. Therefore, the detection of lytic bone lesions on imaging, especially CT and MRI, is becoming crucial from the clinical viewpoint to separate asymptomatic from symptomatic MM patients and the detection of focal lytic lesions is becoming relevant even when no clinical symptoms are present [14]. In a recent report, it has been highlighted by Hillengass [15] that bone imaging in $\mathrm{MM}$ is important for diagnosis due to the fact that osteolytic lesions are a reason for treatment. Medical imaging is clearly crucial for localization of bone pain and prevention of complications such as pathologic fractures on long bones such as the femur and vertebral collapse. According to Hillengass [15], bone marrow imaging can identify focal lesions which is related to the risk of progression. From the prognostic point of view, it has to be remembered that the presence of a focal lesion detected with imaging is a strong prognostic marker requiring prompt treatment even in the absence of bone destruction. In recent years, the major development acquired in $\mathrm{MM}$ imaging is the replacement of standard radiography with CT. Indeed, a study made by the International Myeloma Working Group found lytic lesions using CT on $25 \%$ of patients with a negative radiograph [4]. Using MRI, different bone marrow infiltration patterns could be detected: minimal, diffuse, focal, or mixed focal and diffuse. Bone marrow features in MM are clinically relevant because random bone marrow samples of the pelvis may miss a pathologic bone [4]. Detection of osteoporosis in patients with MM using MRI can be useful to separate pathologic fractures from benign osteoporotic fractures, especially using advanced MRI sequences such as diffusion-weighted sequences. PET/CT imaging is both a functional and morphological technique useful for treatment response assessment; indeed, the disappearance of focal lesions is an important prognostic factor well demonstrated in literature. In MM patients with complete remission, if PET/CT detects the presence of focal lesions, the prognosis is worse. On the contrary, patients with a deep remission after treatment and PET negativity have a very good prognosis if combined with minimal residual disease (MRD) negativity. Finally, potentials of CT are also related to the possibility of distinguishing between MM and other metastatic bone lesions. A recent paper by Uygar Mutlu et al. [16] evaluated, with CT, 320 lesions of 207 patients with MM or metastasis using biopsy or clinical examination as ground truth. It was found that high-density areas on CT were more common in metastasis than MM lesions ( $85.2 \%$ versus $19 \%$ ). Other radiological features differentiated metastasis from MM. These features were: perilesional sclerosis, heterogeneity and ill-defined margins. CT image analysis revealed that high-density areas inside the lesion increased the probability of a metastasis 25 -fold [16]. In 2014, the diagnostic criteria for multiple myeloma were updated by international reference organizations. We report here the key point raised by the revised recommendations and consensus [17-19]:

- Whole-body low-dose CT is recommended by international reference organizations for detection of lytic bone lesions;

- $\quad$ Focal myeloma lesions detected on whole-body MRI will indicate symptomatic multiple myeloma requiring therapy;

- The IMWG recommends using cross-sectional imaging in the initial work-up: wholebody low-dose CT, MRI, or PET/CT, depending on availability and resources.

18F-FDG-PET/CT could be included in the definition of minimal residual disease after therapy due to its functional capabilities. Wide availability of 18F-FDG-PET/CT 
is a potential drawback. Nowadays, radiology is pivotal in the staging and accurate management of patients with MM even in early phases of the disease. Medical imaging is used not only to detect bone lesions but also to predict the risk of early progression from smoldering MM (SMM) to active MM and to identify extra-medullary disease $[2,12,14]$. $\mathrm{CT}$ is particularly useful in identifying the sites of either possible pathologic fractures or neurologic complications and to score bone damage quantitatively [7,12]. Further, compared to conventional radiography, PET/CT and whole-body low-dose CT (WBLDCT) are able to detect the presence of active disease in up to $25 \%$ to $40 \%$ of cases negative at conventional radiography [2]. Following this strictly evidence-based approach, a grade $\mathrm{A}$ recommendation has therefore been assigned to the incorporation of these new imaging modalities (WBLDCT and PET/CT) [7,9,10].

Yet, the diagnostic and prognostic capabilities of medical imaging in MM are still under investigation and development. In fact, significant variability in image-based prognostic scores is present among different centers and in clinical practice $[5,11,13,20,21]$. Further, although the updated version of the IMWG criteria accepts the use of CT, WBLDCT and $\mathrm{PET} / \mathrm{CT}$ to diagnose lytic bone disease in MM, there is still a lack of reliable computational tools for increasing the prognostic value of these modern imaging modalities. In the present review, we will briefly describe the role of new radiological achievements to increase diagnostic potential of medical imaging, with a specific focus on CT.

\section{Quantitative Evaluation of Bone CT and Reader's Experience}

As illustrated by a vast amount of scientific literature, daily clinical practice presents a wide usage of CT data for the diagnosis and follow-up of patients with MM, but, at the same time, there is high variability in image interpretation due to different factors $[3-5,11-14,22,23]$. For instance, it is not always possible to obtain WBLDCT in every patient and often patients with MM receive standard total body CT including thorax and abdominal evaluations. However, after standard reporting of thorax and abdominal findings, in patients affected by MM the focus should be given to small lytic lesions [3-5,24]. Further, the largest number of $\mathrm{CT}$ examinations of patients affected by MM are performed on the elderly, which implies that multiple degenerative bone changes are likely to influence the radiological report reducing the agreement among readers in CT image interpretation to detect even clinically significant small lytic lesions $[6,8,10,11]$. As a consequence, it is probably correct to state that quantitative evaluation of $\mathrm{MM}$ is already feasible by relying on either the multiparametric imaging approach provided by MRI or the functional one provided by PET, but that this same quantitative assessment based just on $\mathrm{CT}$ investigation is still an open issue. As a confirmation of this, an interesting study focused on the comparison of qualitative and quantitative MRI and CT parameters for assessing the involvement of the axial skeleton in MM patients and came to the conclusion that there was no actual benefit from using quantitative parameters for both imaging modalities [25].

As an operational attempt to overcome this deadlock, in order to quantitatively evaluate the status of bone damage, risk of fracture and instability in MM, to reduce reader's variability, and to assess CT data with good agreement, radiologists and clinicians have developed the Myeloma Spine and Bone Damage Score (MSBDS) [26].

The MSBDS scoring system presents several positive aspects:

- On a series of 70 patients with total body CT available and acquired at the same center, the MSBDS criteria resulted as being fast, reproducible and easy to integrate in daily clinical practice;

- $\quad$ MSBDS resulted to be useful not only for radiologists specifically trained to assess the musculoskeletal system, but also for clinicians with no formal training in radiology [26];

- MSBDS correlated well with other quantitative evaluation systems such as the MYRADS score, supporting the reliability of the MSBDS criteria and suggesting that this scoring system could be reliable for total-body CT in MM patients; 
- $\quad$ MSBDS has the unique feature of being specifically designed and tailored to MM patients while, on the contrary, previously published scoring systems developed mainly in orthopedic environments were designed for spinal assessment in metastatic patients [27];

- MSBDS not only evaluates the bones to look for spinal instability, but the lytic bony damage is considered a prognostic target. Specific items of MSBDS are dedicated to the proximal femur involvement and to lytic lesions;

- $\quad$ MSBDS could be far more reliable and diffuse than other scores used for MM patients such as the MY-RADS score and the IMPeTUs criteria for PET or PET/CT [13].

- At a very practical level, MSDBS can be used on CT images that are far more available than MR images, is very fast and easily reproducible and requires the scoring of a low number of parameters.

The Durie-Salmon System and the International Staging System are still the standard of care in patients with MM for staging. However, the increasing number of patients with MM evaluated with CT needs a thorough evaluation with reliable and quantitative parameters as suggested by recent advancement in precision medicine $[28,29]$. The MSDBS is ready for immediate clinical application and improves current methods of scoring bone lesions in MM (Table 1). We also point out that MSBDS is only an option to quantitatively score MM bone involvement and it should represent a starting point to correctly evaluate the patient's impairment not only in clinical practice but also in the medico-legal field (e.g., private health insurances). Every quantitative criteria and potential imaging biomarker must be validated in large trials. At the present time, a prospective clinical validation of MSBDS criteria is underway and results will be available as soon as negative effects of the COVID-19 pandemic are less severe on radiological research [30].

Table 1. Myeloma Spine and Bone Damage Score (MSBDS). Interpretation: High risk: >10: immediate surgical or radiation oncologist consultation. Medium risk: $\geq 5-10$ : possible instability and medium risk of pathologic fracture. Low risk: $<5 .{ }^{*}$ Bone abnormalities not sufficient to give high risk scores, if isolated. ${ }^{* *} 1$ point for every segment according to MY-RADS (from [26]).

\begin{tabular}{cc}
\hline Location & Points \\
\hline Junctional Spine (C0-C2, C7-T2, T11-L1, L5-S1) & 3 \\
Mobile Spine (C3-C6, L2-L4) & 2 \\
Collapse/involvement $>50 \%$ & 3 \\
Collapse $<50 \%$ * & 2 \\
Posterolateral (facet, pedicle) involvement monolateral & 2 \\
Posterolateral (facet, pedicle) bilateral monolateral & 3 \\
Spinal Canal involvement & 5 \\
Trochanteric region focal lesions < $10 \mathrm{~mm}$ & 2 \\
Femoral neck or entire trochanteric region & 5 \\
More 2/3 of bone diameter & 3 \\
Focal lesion > 5 mm at any site * & 1 \\
Diffuse Pattern & 1 **
\end{tabular}

Another important issue in medical imaging related to MM is concerned with the reader's experience. Radiology is a wide field where different subspecialties are present. For example, the European Society of Radiology, who promotes and coordinates the scientific, philanthropic, intellectual and professional activities of radiology, have several affiliated subspecialties, reflecting great heterogeneity in radiological profession [31]. For $\mathrm{MM}$, the interpretation of bony lesions is straightforward in most cases due to the fact that lesions are lytic. However, in some cases, lesion could be numerous, well circumscribed and could punch out lucencies, raindrop skull, endosteal scalloping and sometimes generalized osteopenia [32]. In these cases, it is likely that a subspecialty with experience in reading CT and MRI data will enhance the role of CT and MRI for MM [5,26]. In many centers, consultation and second-opinion interpretation of medical images by subspecialty radiologists are routinely performed [5,33-37]. A recent study was conducted with the aim of improv- 
ing the radiological detection and characterization of clinically significant lytic lesions using standard CT (Table 2). Discrepancy rates up to $15 \%$ have been reported, describing reports by radiologists at different levels of training and radiologists at different clinical settings, while the discrepancy rate in interpreting a clinically important abnormality (e.g., interpreting the presence of a lytic lesion $>5 \mathrm{~mm}$ ) reached $21 \%$ [5]. A clinical benefit of a subspecialty second-opinion consultation in MM CT has been demonstrated, in particular, for lytic lesions. Indeed, a lytic lesion in MM is sometimes difficult to detect, especially when the diameter is between 5 and $10 \mathrm{~mm}$ and when it is located in an osteoporotic and degenerated vertebral body. Particularly for these patients, dedicated musculoskeletal (MSK) radiologists could solve difficult and uncertain cases $[5,36,38]$. The expertise of a dedicated reader is also crucial because a reference standard is difficult to achieve in MM since bone biopsy cannot be obtained in small lesions and in every anatomical location.

Table 2. Example of minimal computed tomography technical parameters for lytic lesion detection in multiple myeloma (from: [5]).

\begin{tabular}{|c|c|}
\hline Number of Detector Rows & 16 or More up to 128 \\
\hline Minimum Scan coverage & Skull base to femur \\
\hline Tube voltage $(\mathrm{kV}) /$ time-current product (mAs) & 120/50-70, adjusted as clinically needed \\
\hline Reconstruction convolution kernel & $\begin{array}{l}\text { Sharp, high-frequency (bone) and smooth (soft } \\
\text { tissue). Middle-frequency kernel for all images } \\
\text { are adjusted by the radiologist as } \\
\text { deemed necessary }\end{array}$ \\
\hline Iterative reconstruction algorithms & Yes (to reduce image noise and streak artefacts) \\
\hline Thickness & $\leq 5 \mathrm{~mm}$ \\
\hline Multiplanar Reconstructions (MPRs) & $\begin{array}{l}\text { Yes (sagittal, coronal and parallel to long axis } \\
\text { of proximal limbs) }\end{array}$ \\
\hline Matrix, Rotation time, table speed, pith index & $128 \times 128,0.5 \mathrm{~s}, 24 \mathrm{~mm}$ per gantry rotation, 0.8 \\
\hline
\end{tabular}

\section{Radiomics in MM}

Radiomics is a very recent approach to the diagnostic, prognostic and therapeutic assessment of specific diseases, which, in its most recent variation, relies on the use of pattern recognition for the extraction of quantitative descriptors from imaging data (typically acquired by means of structural modalities) and on their use with prediction purposes by means of computational algorithms based on Artificial Intelligence (AI) [39-43].

Radiomics is performed using dedicated software on standard radiological images. Interestingly enough, a quick search on most databases immediately points out that the number of papers dealing with the use of AI-based radiomics as applied to MM is up to one order of magnitude smaller than the number of papers devoted to the application of this same technique to breast cancer data ([44] and references therein) and even two orders of magnitude smaller than in the case of lung cancer [45] and references therein. The reason for this dramatic difference is probably in the notable genetic heterogeneity of $\mathrm{MM}$, which has notable impacts on the pathogenesis and progression of the disease. In addition, few articles were published in the early 2012/2013 dealing with radiomics and radiology; in the year 2020, it was possible to find more than 1000 articles on PubMed (https: / pubmed.ncbi.nlm.nih.gov/?term=\%28radiomics $\% 29 \% 29+$ AND+\%2 8radiology\%29\&filter=years.2020-2021) reflecting the growing interest in this field. As stated in 2012 by Lambin et al. [40], development of radiomics comes from the potential to overcome some known limitations of bioptical samples in cancer. Indeed, solid cancers, including MM for bone involvement, are characterized by an extraordinarily spatial and temporal heterogeneity involving genes, proteins, cells, microenvironment and tissues. Radiomics could be considered a kind of noninvasive imaging tool able to study and define intratumoural heterogeneity using only data included in medical images and without the 
need or limitations of a soft-tissue or bone biopsy. From 2012 to 2021, a wide amount of scientific research has been made regarding radiomics and other tools related to the artificial intelligence fields have been adopted. Machine learning and deep learning are strongly associated with radiomics workflow. Machine learning includes computational algorithms using the image features extracted by radiomics as the input in order to provide predictions concerning disease outcomes on follow-up as the output. In MM patients, radiomics and machine learning can be used to predict outcomes on the basis of MRI or CT imaging features. Machine learning can be unsupervised if no information is provided by or determined by an available historical set of data. Machine learning is supervised if machine learning methods are trained with an available data archive. Finally, machine learning with deep learning uses imaging input into a multilayer neural network to sequentially modify the target and reduce its size until a set of numbers is automatically produced.

Indeed, MM is a genetically complex disease that evolves from premalignant stages, such as M-GUS and SMM, and progresses to symptomatic MM [46].

In $\mathrm{MM}$, the development of the disease is very complex and a progression with clonal sweeps in the early phase and a regional evolution in advanced disease have been recently confirmed [6]. Therefore, an analysis of multiple bone lesions could be performed using computer algorithms supporting the use of radiomics. For instance, a recent application of radiomics in $\mathrm{MM}$ showed that, in clinical practice, radiomics is able to improve the radiological evaluation of focal and diffuse pattern of MM on CT by improving the Area Under the Curve (AUC) of radiologists [47]. Specifically, accuracy in terms of the AUC of radiologists compared to the reference standard was lower (64\%) than accuracy computed using a radiomics approach, which obtained a maximum value of $79 \%$. Further, by using a radiomics approach it is possible to increase the reading accuracy of radiological characterization of focal and diffuse pattern of MM on standard CT [47].

With specific reference to $\mathrm{CT}$ data, the use of $\mathrm{AI}$ for the assessment of MM radiomics typically relies on the two-step process illustrated in Figure 1:

1. Pattern recognition and property extraction algorithms [21,44] is applied to either specific bone lesions or the whole skeleton asset in order to extract quantitative descriptors of the impact of the disease on the MM bone structure;

2. Machine learning [44,48], in either its unsupervised or supervised version, is applied against the descriptors extracted by step 1 in order to both stratify the MM patients on the basis of their CT data characteristics and predict the disease outcome as far as post-transplantation relapse is concerned.

Preliminary results obtained by means of this kind of approach [48] show that MM is associated with an extension of the intrabone volume for the whole body and that machine learning can identify CT image properties mostly correlating with the disease evolution.

However, the use and validation of radiomics for prognostic purposes in $\mathrm{MM}$ is still in progress and several factors have to be considered before assuming radiomics results are completely reliable, repeatable and feasible in clinical practice. In general, medical imaging is capable of generating imaging biomarkers, while acquisition and analysis processes are different from frequently used comparators such as blood or urine biomarkers. This difference is related to the methodology for obtaining the sample. In radiology, the acquisition of the sample (i.e., the data set of images) is heterogeneous by design, since complex equipment from different vendors is clinically used. Even with multiple human and technological efforts, standardization of image quality as an input for different imaging biomarkers analysis is difficult and it is not yet clear whether a complete standardization can be realized. Several scientific societies such as the European Society of Radiology and the Radiological Society of North America (RSNA) tried to provide standards for the best possible standardization at the acquisition level and the minimum requirements for the image analysis software used in the qualification of imaging biomarkers [28,29,41,48-50]. However, more sophisticated corrective measures could be taken by artificial intelligence (AI)-based approaches to let complex and deep neural 
networks learn from the lack of homogeneity in the collected images, both in the DICOM metadata and in the pixel information, and adjust the imaging parameters to be analyzed.
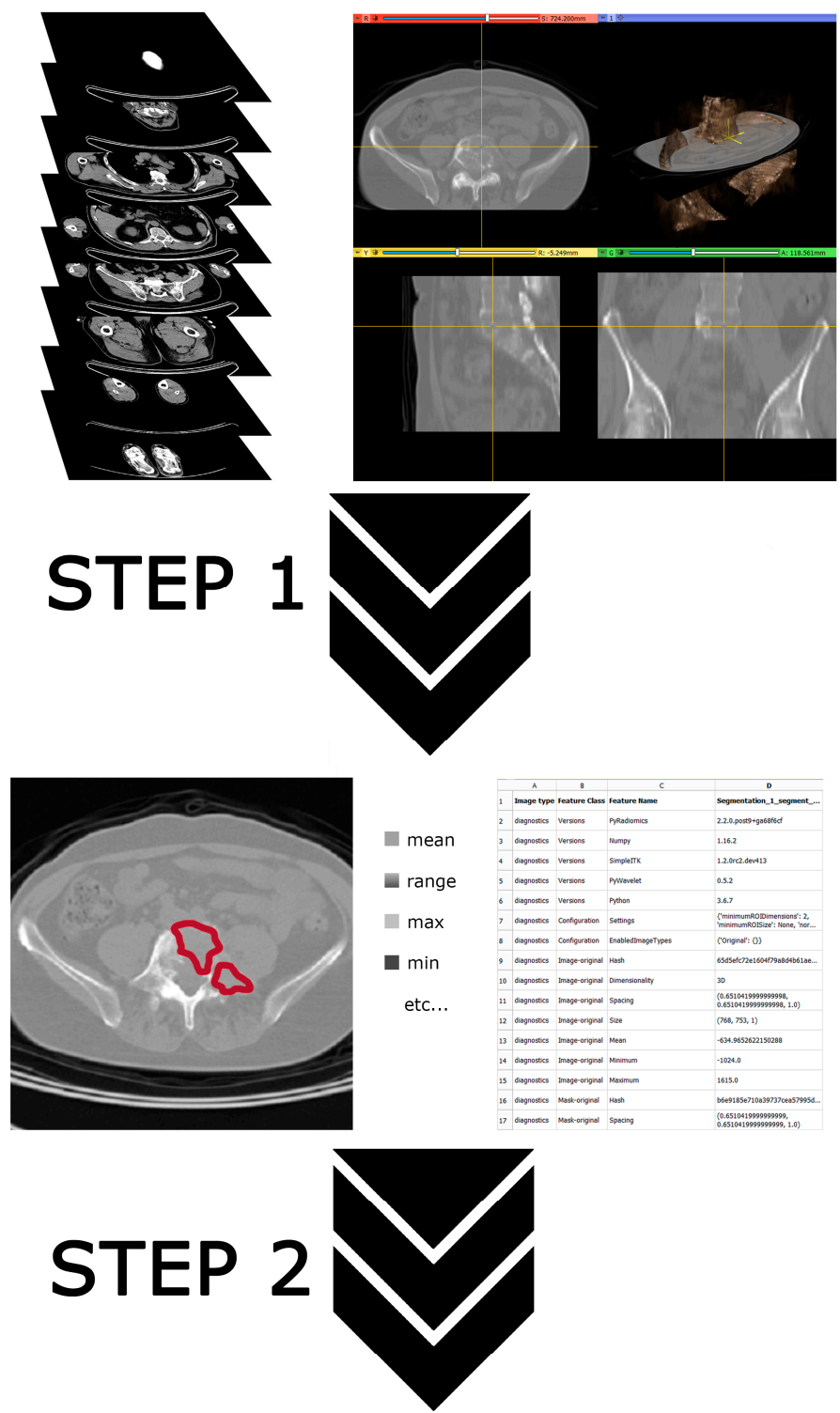

Clustering on radiomics features

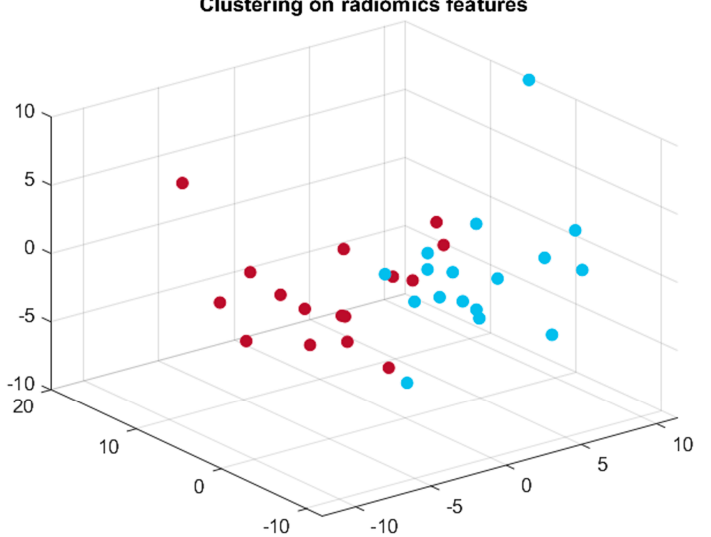

Figure 1. The two-step process of Artificial Intelligence (AI)-based radiomics. The input data (first row) are fed into a property extraction algorithm to obtain a set of image features (second row). These features are fed into a machine learning algorithm to obtain patients' stratification (third row). 


\section{Conclusions}

There is still high variability in the choice between various imaging methods to study MM patients and high variability in image interpretation with suboptimal agreement among readers even in tertiary centers. Therefore, the potential of medical imaging for patients affected by MM is still to be completely unveiled. In the coming years, new insights to study MM with medical imaging will derive from AI and radiomics usage in different bone lesions and from the wide implementations of quantitative methods to report CT and MRI. Eventually, medical imaging data can be integrated with the patient's outcomes, with the purpose of finding radiological biomarkers for predicting the prognostic flow and therapeutic response of the disease.

Author Contributions: Conceptualization, A.S.T. and M.P.; methodology, all authors; writingoriginal draft preparation, all authors; writing — review and editing, all authors. All authors have read and agreed to the published version of the manuscript.

Funding: This research this work was partially supported by grants from Italian Ministry of Health (Ricerca Corrente).

Institutional Review Board Statement: Not applicable.

Informed Consent Statement: Not applicable.

Conflicts of Interest: The authors declare no conflict of interest.

\section{References}

1. Jameson, J.L.; Fauci, A.S.; Kasper, D.L.; Hauser, S.L.; Longo, D.L. Harrison's Principles of Internal Medicine. JAMA J. Am. Med. Assoc. 2018. [CrossRef]

2. Zamagni, E.; Cavo, M.; Fakhri, B.; Vij, R.; Roodman, D. Bones in Multiple Myeloma: Imaging and Therapy. Am. Soc. Clin. Oncol. Educ. Book 2018, 38, 638-646. [CrossRef] [PubMed]

3. Tagliafico, A.S.; Cea, M.; Rossi, F.; Valdora, F.; Bignotti, B.; Succio, G.; Gualco, S.; Conte, A.; Dominietto, A. Differentiating diffuse from focal pattern on Computed Tomography in multiple myeloma: Added value of a Radiomics approach. Eur. J. Radiol. 2019. [CrossRef]

4. Hillengass, J.; Moulopoulos, L.A.; Delorme, S.; Koutoulidis, V.; Mosebach, J.; Hielscher, T.; Drake, M.; Rajkumar, S.V.; Oestergaard, B.; Abildgaard, N.; et al. Whole-body computed tomography versus conventional skeletal survey in patients with multiple myeloma: A study of the International Myeloma Working Group. Blood Cancer J. 2017, 7, e599. [CrossRef] [PubMed]

5. Tagliafico, A.S.; Belgioia, L.; Bonsignore, A.; Rossi, F.; Succio, G.; Bignotti, B.; Dominietto, A. Subspecialty second-opinion in multiple myeloma ct: Emphasis on clinically significant lytic lesions. Medicina 2020, 56, 195. [CrossRef]

6. Rasche, L.; Angtuaco, E.J.; Alpe, T.L.; Gershner, G.H.; McDonald, J.E.; Samant, R.S.; Kumar, M.; Van Hemert, R.; Epstein, J.; Deshpande, S.; et al. The presence of large focal lesions is a strong independent prognostic factor in multiple myeloma. Blood 2018. [CrossRef]

7. Rajkumar, S.V. Evolving diagnostic criteria for multiple myeloma. Hematology 2015. [CrossRef]

8. Hillengass, J.; Fechtner, K.; Weber, M.-A.; Bäuerle, T.; Ayyaz, S.; Heiss, C.; Hielscher, T.; Moehler, T.M.; Egerer, G.; Neben, K.; et al. Prognostic Significance of Focal Lesions in Whole-Body Magnetic Resonance Imaging in Patients with Asymptomatic Multiple Myeloma. J. Clin. Oncol. 2010, 28, 1606-1610. [CrossRef]

9. Ekert, K.; Hinterleitner, C.; Baumgartner, K.; Fritz, J.; Horger, M. Extended texture analysis of non-enhanced whole-body mri image data for response assessment in multiple myeloma patients undergoing systemic therapy. Cancers 2020, 12, 761. [CrossRef]

10. Moulopoulos, L.A.; Koutoulidis, V.; Hillengass, J.; Zamagni, E.; Aquerreta, J.D.; Roche, C.L.; Lentzsch, S.; Moreau, P.; Cavo, M.; Miguel, J.S.; et al. Recommendations for acquisition, interpretation and reporting of whole body low dose CT in patients with multiple myeloma and other plasma cell disorders: A report of the IMWG Bone Working Group. Blood Cancer J. 2018, 8, 95. [CrossRef]

11. Rossi, F.; Torri, L.; Dominietto, A.; Tagliafico, A.S. Spectrum of magnetic resonance imaging findings in transplanted multiple myeloma patients with hip/pelvic pain (according to MY-RADS): A single center experience. Eur. J. Radiol. 2020. [CrossRef]

12. Rajkumar, S.V.; Dimopoulos, M.A.; Palumbo, A.; Blade, J.; Merlini, G.; Mateos, M.-V.; Kumar, S.; Hillengass, J.; Kastritis, E.; Richardson, P.; et al. International Myeloma Working Group updated criteria for the diagnosis of multiple myeloma. Lancet Oncol. 2014, 15, e538-e548. [CrossRef]

13. Nanni, C.; Versari, A.; Chauvie, S.; Bertone, E.; Bianchi, A.; Rensi, M.; Bellò, M.; Gallamini, A.; Patriarca, F.; Gay, F.; et al. Interpretation criteria for FDG PET/CT in multiple myeloma (IMPeTUs): Final results. IMPeTUs (Italian myeloma criteria for PET USe). Eur. J. Nucl. Med. Mol. Imaging 2018, 45, 712-719. [CrossRef] 
14. Messiou, C.; Hillengass, J.; Delorme, S.; Lecouvet, F.E.; Moulopoulos, L.A.; Collins, D.J.; Blackledge, M.D.; Abildgaard, N.; Østergaard, B.; Schlemmer, H.-P.; et al. Guidelines for acquisition, interpretation, and reporting of whole-body MRI in myeloma: Myeloma response assessment and diagnosis system (MY-RADS). Radiology 2019. [CrossRef]

15. Hillengass, J. Evolving Concepts in the Diagnosis and Staging of Multiple Myeloma. Natl. Compr. Cancer Netw. 2020, 18, 1770-1772. [CrossRef]

16. Mutlu, U.; Balci, A.; Özsan, G.H.; Özkal, S.; Şeyhanli, A.; Özgül, H.A. Computed tomography characteristics of multiple myeloma and other osteolytic metastatic bone lesions. Acta Radiol. SAGE Publ. 2020. [CrossRef]

17. Mosebach, J.; Thierjung, H.; Schlemmer, H.-P.; Delorme, S. Multiple Myeloma Guidelines and Their Recent Updates: Implications for Imaging. Rofo Geb. Rontgenstr. Nukl. 2019, 191, 998-1009. [CrossRef]

18. Moreau, P.; Attal, M.; Caillot, D.; Macro, M.; Karlin, L.; Garderet, L.; Facon, T.; Benboubker, L.; Escoffre-Barbe, M.; Stoppa, A.-M.; et al. Prospective Evaluation of Magnetic Resonance Imaging and [18F]Fluorodeoxyglucose Positron Emission TomographyComputed Tomography at Diagnosis and Before Maintenance Therapy in Symptomatic Patients With Multiple Myeloma Included in the IFM/DFCI 2009 Trial: Results of the IMAJEM Study. J. Clin. Oncol. Off. J. Am. Soc. Clin. Oncol. 2017, 35, $2911-2918$. [CrossRef]

19. Palumbo, A.; Avet-Loiseau, H.; Oliva, S.; Lokhorst, H.M.; Goldschmidt, H.; Rosinol, L.; Richardson, P.; Caltagirone, S.; Lahuerta, J.J.; Facon, T.; et al. Revised International Staging System for Multiple Myeloma: A Report from International Myeloma Working Group. J. Clin. Oncol. Off. J. Am. Soc. Clin. Oncol. 2015, 33, 2863-2869. [CrossRef]

20. Martinoli, C.; Bacigalupo, L.; Forni, G.L.; Balocco, M.; Garlaschi, G.; Tagliafico, A. Musculoskeletal manifestations of chronic anemias. Semin. Musculoskelet Radiol. 2011, 15. [CrossRef]

21. Schenone, D.; Lai, R.; Cea, M.; Rossi, F.; Torri, L.; Bignotti, B.; Succio, G.; Gualco, S.; Conte, A.; Dominietto, A.; et al. Radiomics and artificial intelligence analysis of $\mathrm{CT}$ data for the identification of prognostic features in multiple myeloma. Med. Imaging 2020, $11314,113144 \mathrm{~A}$.

22. Reinert, C.P.; Krieg, E.M.; Bösmüller, H.; Horger, M. Mid-term response assessment in multiple myeloma using a texture analysis approach on dual energy-CT-derived bone marrow images a proof of principle study. Eur. J. Radiol. 2020. [CrossRef] [PubMed]

23. Kosmala, A.; Weng, A.M.; Krauss, B.; Knop, S.; Bley, T.A.; Petritsch, B. Dual-energy CT of the bone marrow in multiple myeloma: Diagnostic accuracy for quantitative differentiation of infiltration patterns. Eur. Radiol. 2018, 28, 5083-5090. [CrossRef] [PubMed]

24. Kobayashi, H.; Abe, Y.; Narita, K.; Kitadate, A.; Takeuchi, M.; Matsue, K. Prognostic significance of medullary abnormalities of the appendicular skeleton detected by low-dose whole-body multidetector computed tomography in patients with multiple myeloma. Blood 2017, 130, 1763.

25. Horger, M.; Fritz, J.; Thaiss, W.M.; Ditt, H.; Weisel, K.; Haap, M.; Kloth, C. Comparison of qualitative and quantitative CT and MRI parameters for monitoring of longitudinal spine involvement in patients with multiple myeloma. Skelet. Radiol. 2018, 47, 351-361. [CrossRef] [PubMed]

26. Tagliafico, A.S.; Belgioia, L.; Bonsignore, A.; Signori, A.; Formica, M.; Rossi, F.; Piana, M.; Schenone, D.; Dominietto, A. Development and definition of a simplified scoring system in patients with multiple myeloma undergoing stem cells transplantation on standard computed tomography: Myeloma spine and bone damage score (MSBDS). Cancer Imaging 2020, 20. [CrossRef] [PubMed]

27. Fisher, C.G.; DiPaola, C.P.; Ryken, T.C.; Bilsky, M.H.; Shaffrey, C.I.; Berven, S.H.; Harrop, J.S.; Fehlings, M.G.; Boriani, S.; Chou, D.; et al. A novel classification system for spinal instability in neoplastic disease: An evidence-based approach and expert consensus from the spine oncology study group. Spine 2010. [CrossRef] [PubMed]

28. European Society of Radiology (ESR). Medical imaging in personalised medicine: A white paper of the research committee of the European Society of Radiology (ESR). Insights Imaging 2015, 6, 141-155. [CrossRef] [PubMed]

29. European Society of Radiology (ESR). ESR Statement on the Validation of Imaging Biomarkers. Insights Imaging 2020. [CrossRef]

30. European Society of Radiology (ESR). Impact of coronavirus disease 2019 (COVID-19) emergency on Italian radiologists: A national survey. Eur. Radiol. 2020. [CrossRef]

31. European Society of Radiology (ESR); American College of Radiology (ACR). European Society of Radiology (ESR) and American College of Radiology (ACR) report of the 2015 global summit on radiological quality and safety. Insights Imaging 2016. [CrossRef]

32. Lalam, R.; Bloem, J.L.; Noebauer-Huhmann, I.M.; Wörtler, K.; Tagliafico, A.; Vanhoenacker, F.; Nikodinovska, V.V.; Sanal, H.T.; van der Woude, H.-J.; Papakonstantinou, O.; et al. ESSR Consensus Document for Detection, Characterization, and Referral Pathway for Tumors and Tumorlike Lesions of Bone. Semin. Musculoskelet Radiol. 2017, 21, 630-647. [CrossRef] [PubMed]

33. Löfgren, J.; Loft, A.; Barbosa de Lima, V.A.; Østerlind, K.; von Benzon, E.; Højgaard, L. Clinical importance of re-interpretation of PET/CT scanning in patients referred to a tertiary care medical centre. Clin. Physiol. Funct. Imaging 2017, 37, 143-147. [CrossRef] [PubMed]

34. Hatzoglou, V.; Omuro, A.M.; Haque, S.; Khakoo, Y.; Ganly, I.; Oh, J.H.; Shukla-Dave, A.; Fatovic, R.; Gaal, J.; Holodny, A.I. Second-opinion interpretations of neuroimaging studies by oncologic neuroradiologists can help reduce errors in cancer care. Cancer 2016, 122, 2708-2714. [CrossRef]

35. Lakhman, Y.; D'Anastasi, M.; Miccò, M.; Scelzo, C.; Vargas, H.A.; Nougaret, S.; Sosa, R.E.; Chi, D.S.; Abu-Rustum, N.R.; Hricak, H.; et al. Second-Opinion Interpretations of Gynecologic Oncologic MRI Examinations by Sub-Specialized Radiologists Influence Patient Care Conclusions-Expert second-opinion review of GynOnc MRI influences patient care. HHS Public Access. Eur. Radiol. 2016, 26, 2089-2098. [CrossRef] 
36. Chalian, M.; Del Grande, F.; Thakkar, R.S.; Jalali, S.F.; Chhabra, A.; Carrino, J.A. Second-opinion subspecialty consultations in musculoskeletal radiology. Am. J. Roentgenol. 2016, 206, 1217-1221. [CrossRef]

37. Rozenberg, A.; Kenneally, B.E.; Abraham, J.A.; Strogus, K.; Roedl, J.B.; Morrison, W.B.; Zoga, A.C. Second opinions in orthopedic oncology imaging: Can fellowship training reduce clinically significant discrepancies? Skeletal Radiol. Skelet. Radiol. 2019, 48, 143-147. [CrossRef]

38. Snoj, Ž.; Hebar, T.; Sconfienza, L.M.; Vanhoenacker, F.M.H.M.; Shahabpour, M.; Salapura, V.; Isaac, A.; Drakonaki, E.; Vasilev, Y.; Drape, J.-L.; et al. Present Status of Musculoskeletal Radiology in Europe: International Survey by the European Society of Musculoskeletal Radiology. Semin. Musculoskelet Radiol. 2020. [CrossRef]

39. Gillies, R.J.; Kinahan, P.E.; Hricak, H. Radiomics: Images Are More than Pictures, They Are Data. Radiology 2016, $278,563-577$. [CrossRef]

40. Lambin, P.; Rios-Velazquez, E.; Leijenaar, R.; Carvalho, S.; van Stiphout, R.G.P.M.; Granton, P.; Zegers, C.M.L.; Gillies, R.; Boellard, R.; Dekker, A.; et al. Radiomics: Extracting more information from medical images using advanced feature analysis. Eur. J. Cancer Oxf. Eng. 2012, 48, 441-446. [CrossRef]

41. Lambin, P.; Leijenaar, R.T.H.; Deist, T.M.; Peerlings, J.; de Jong, E.E.C.; van Timmeren, J.; Sanduleanu, S.; Larue, R.T.H.M.; Even, A.J.G.; Jochems, A.; et al. Radiomics: The bridge between medical imaging and personalized medicine. Nat. Rev. Clin. Oncol. 2017, 14, 749-762. [CrossRef] [PubMed]

42. Parmar, C.; Grossmann, P.; Bussink, J.; Lambin, P.; Aerts, H.J.W.L. Machine Learning methods for Quantitative Radiomic Biomarkers. Sci. Rep. Nat. Publ. Group 2015, 5, 13087. [CrossRef] [PubMed]

43. Yip, S.S.F.; Aerts, H.J.W.L. Applications and limitations of radiomics. Phys. Med. Biol. 2016, 61, R150-R166. [CrossRef] [PubMed]

44. Tagliafico, A.S.; Piana, M.; Schenone, D.; Lai, R.; Massone, A.M.; Houssami, N. Overview of radiomics in breast cancer diagnosis and prognostication. Breast 2020. [CrossRef] [PubMed]

45. Kothari, G.; Korte, J.; Lehrer, E.J.; Zaorsky, N.G.; Lazarakis, S.; Kron, T.; Hardcastle, N.; Siva, S. A systematic review and meta-analysis of the prognostic value of radiomics based models in non-small cell lung cancer treated with curative radiotherapy. Radiother. Oncol. J. Eur. Soc. Radiol. Oncol. 2020, 155, 188-203. [CrossRef]

46. Manier, S.; Salem, K.Z.; Park, J.; Landau, D.A.; Getz, G.; Ghobrial, I.M. Genomic complexity of multiple myeloma and its clinical implications. Nat. Rev. Clin. Oncol. 2017. [CrossRef] [PubMed]

47. Fiz, F.; Marini, C.; Piva, R.; Miglino, M.; Massollo, M.; Bongioanni, F.; Morbelli, S.; Bottoni, G.; Campi, C.; Bacigalupo, A.; et al. Adult Advanced Chronic Lymphocytic Leukemia: Computational Analysis of Whole-Body CT Documents a Bone Structure Alteration. Radiology. Radiol. Soc. N. Am. 2014, 271, 805-813. [CrossRef]

48. Rizzo, S.; Botta, F.; Raimondi, S.; Origgi, D.; Fanciullo, C.; Morganti, A.G.; Bellomi, M. Radiomics: The facts and the challenges of image analysis. Eur. Radiol. Exp. 2018, 2, 36. [CrossRef]

49. Fedorov, A.; Beichel, R.; Kalpathy-Cramer, J.; Finet, J.; Fillion-Robin, J.-C.; Pujol, S.; Bauer, C.; Jennings, D.; Fennessy, F.; Sonka, M.; et al. 3D Slicer as an image computing platform for the Quantitative Imaging Network. Magn. Reson. Imaging 2012, 30, $1323-1341$. [CrossRef]

50. Parekh, V.; Jacobs, M.A. Radiomics: A new application from established techniques. Expert Rev. Precis. Med. Drug Dev. 2016, 1, 207-226. [CrossRef] 